\title{
MODELING OF QUASICRYSTAL LATTICES WITH A 4-FOLD SYMMETRY AXIS
}

\author{
O. V. Smolyakov \\ Zaporizhzhya National University, Zaporizhzhya, Ukraine \\ *e-mail: asmolyakov972@gmail.com
}

\begin{abstract}
The construction method of a quasilattice with a four-fold rotational symmetry axis is proposed. The described method is based on the recurrent generation of the initial group of lattice points, which are a set of vertices of a square. The aperiodic crystal reciprocal lattice modeling algorithm is analyzed. Used modeling technique is compared with conventional projection approach. The orthogonal basis of a fourdimensional hypercubic lattice is proposed. This lattice produces two-dimensional quasicrystal with a fourfold symmetry axis after it projection on a flat surface. It is shown that the indexation of diffraction pattern of similar quasiperiodic structures can be carry out using 3 integer indexes, which is analogous to the indexing system proposed by Cahn for application to icosahedral quasicrystals.

Keywords: quasicrystals, aperiodic crystals, reciprocal lattice, diffraction.
\end{abstract}

Received 07.11.2018; Received in revised form 2.12.2018; Accepted 21.12.2018

\section{Introduction}

The discovery of quasicrystalline materials has changed the picture of the laws that govern the structural organization of matter. It has been found that symmetry elements incompatible with translational symmetries did not prohibit the existence of a long-range order in a material's structure. A few years after the discovery of the icosahedral quasicrystals (QC) several alloys were created, which had an eight-, ten- and twelve-fold rotational symmetry axis. The discovery of novel materials with prohibited symmetry axis has also created a problem of describing the structure of quasicrystals. The appearance of such problem is attributed to the impossibility of choosing a unit cell, translations of which would allow one to rebuild the whole structure. Second problem was the inability to describe atomic surfaces using 3 integer Miller indices (HKL). Various solutions of this problem have been proposed. There exist several approaches to describing a QC atomic arrangement. These methods rely on space tessellation using a set of polyhedra, which are then decorated with atoms [1-4]. Beside that, the method of higher dimensional projection of periodic structure to the physical space is also widely utilized $[5,6]$. Several papers $[3,7,8]$ also show the amount of allowed crystallographic groups encountered in QC. In many cases the presence of the forbidden rotational symmetry axis presence of the forbidden rotational symmetry axis is considered to be a main indicator of the QC structure. Still, in the works of $[8,9]$ it is shown that the existence of only conventionally allowed symmetry axis does not, by itself, exclude quasicrystalline material structure. Materials that exhibit such property received a name of aperiodic crystals [8]. Thus, the problem of describing such structures needs closer inspection.

\section{Modeling of the reciprocal quasilattice with a four-fold symmetry axis}

In the works [10-12] a method of modeling two-dimensional octagonal, decagonal and dodecagonal structures is proposed. It is based on the recurrent generation of a group of points, which have a symmetrical property of a lattice that is being modeled. The algorithm of a quasilattice construction is the same for octagonal, decagonal and dodecagonal QC, and can be written as recurrent relations:

$$
D_{n}=D_{n-1}+\left\{k^{n-2} \mathbf{q}_{i}\right\} D_{n-1} .
$$

Expression of this type indicates the addition to the group of points denoted as $D_{n-1}$, of 
the same groups displaced from the origin (center of symmetry) by vectors $\pm k^{n-2} \mathbf{q}_{i}$, where $\pm \mathbf{q}_{i}$ are the basis vectors defining the vertices of the initial regular polygon. Generating decagonal structure requires usage of a regular decagon vertices as basis vectors, as well as a parameter (scaling factor) $k=\tau=(1+\sqrt{5}) / 2-$ a number known as the golden section. Let us use an algorithm (1) to generate a two-dimensional reciprocal quasilattice with a four-fold symmetry axis. Parameter $k$ will be chosen the same as it is for decagonal QC. Basis vectors $\pm \mathbf{q}_{i}$ represent vertices of a square. The result of an execution of the algorithm (1) is shown in a Fig. 1. It can be seen that the use of the algorithm proposed for modeling the reciprocal lattice of decagonal quasicrystals makes it possible to obtain an aperiodic lattice with a 4-fold symmetry axis. The density of point's distribution in this aperiodic lattice is uniform as in quasicrystals.

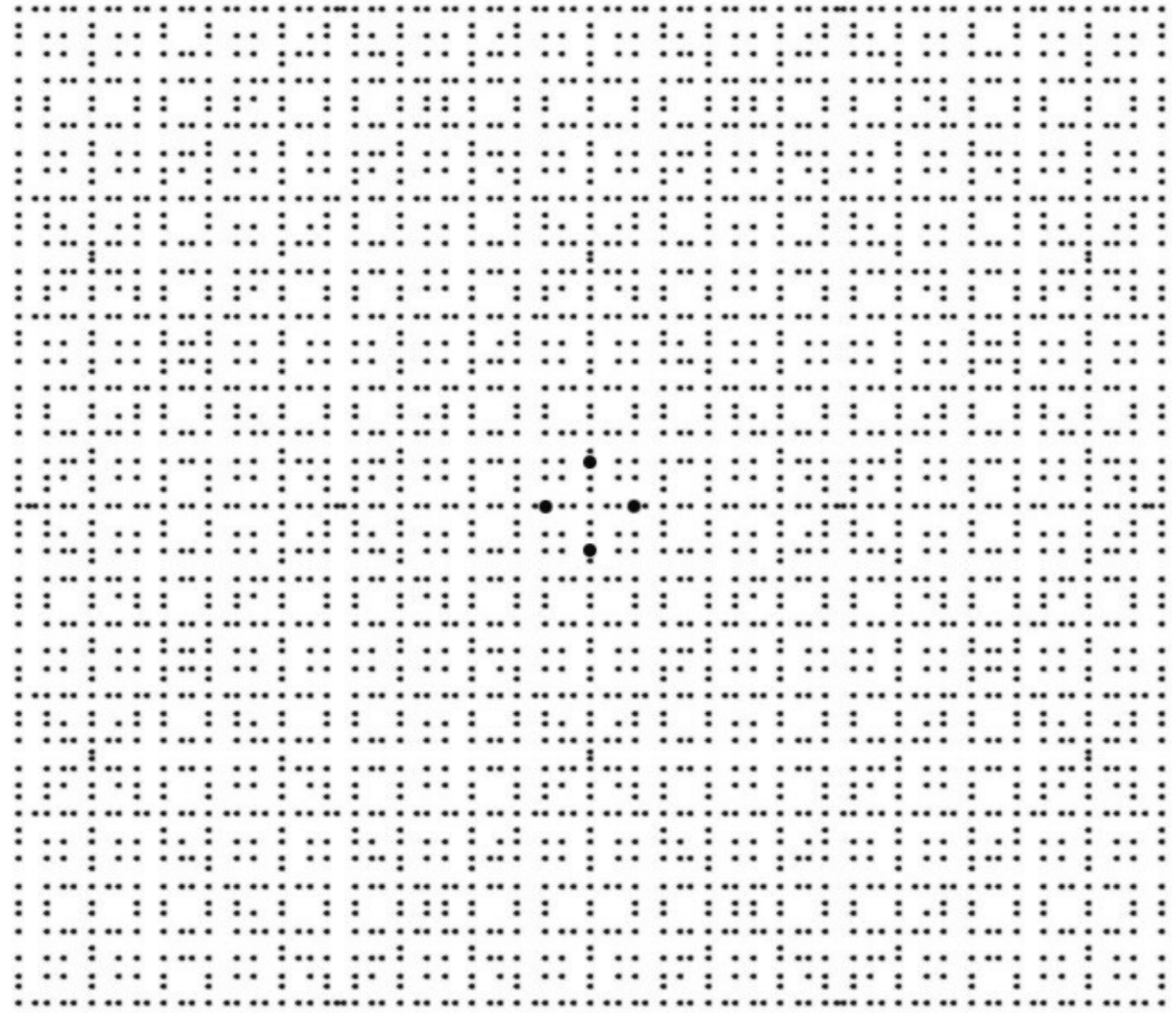

Fig. 1. Quasiperiodic lattice with a four-fold symmetry axis, constructed using the algorithm (1) (marked the initial group of lattice points).

It should be noted that usage of a random number $k$ as a parameter instead of $\tau$ or $\delta_{s}=1+\sqrt{2}$ (silver section) will cause, in generally, an appearance of tears in a structure, or will force the distance between lattice points to approach zero. It was also established that algorithm (1) can be modified in a way that basis vector factors are expressed as a linear combination of aforementioned numbers with integers. 
The higher dimensional periodic lattice projection is one of the most widely used methods for modeling and analyzing quasiperiodic lattices [7]. Let us compare proposed method of recurrent generation from the group of points with conventional projection method. To achieve this, we will use four-dimensional hypercubic lattice with the following basis vectors:

$$
\mathbf{q}_{1}=(1,0, \tau, 0) ; \quad \mathbf{q}_{2}=(0,1,0, \tau) ; \quad \mathbf{q}_{3}=(\tau, 0,-1,0) ; \mathbf{q}_{4}=(0, \tau, 0,-1) .
$$

Each vector among the (2) has two components corresponding to physical space and two components corresponding to perpendicular space. Therefore, these vectors can be written down as $\mathbf{q}_{i}=\left(\mathbf{q}_{i}^{\|} ; \mathbf{q}_{i}^{\perp}\right)$. The type of vectors (2) and the properties of a number $\tau$ force the ability of each lattice point obtained via the usage of the algorithm (1) to be able to be written as a linear combination of the vectors $\mathbf{q}_{i}^{\|}$:

$$
\mathbf{Q}^{\|}=\sum_{1}^{4} n_{i} \mathbf{q}_{i}^{\|}
$$

Each lattice point in physical space has a corresponding vector in a perpendicular space:

$$
\mathbf{Q}^{\perp}=\sum_{1}^{4} n_{i} \mathbf{q}_{i}^{\perp}
$$

As shown in $[7,13]$ the intensity of the diffraction peaks associated with a particular reciprocal lattice point of a $\mathrm{QC}$ is inversely proportional to the distance between the occupation domain of a particular lattice point and the physical space. a form:

It is shown below that squares of a vector modulus of $\mathbf{Q}^{\|}$and $\mathbf{Q}^{\perp}$ can be presented in

$$
\begin{gathered}
\left|\mathbf{Q}^{\|}\right|^{2}=\left(\sum_{1}^{4} n_{i}^{2}\right)+\left(2 n_{1} n_{3}+n_{3}^{2}+2 n_{2} n_{4}+n_{4}^{2}\right) \tau=N+M \tau \\
\left|\mathbf{Q}^{\perp}\right|^{2}=\left(\sum_{1}^{4} n_{i}^{2}\right)+\left(n_{1}^{2}-2 n_{1} n_{3}+n_{2}^{2}-2 n_{2} n_{4}\right) \tau=\tau(N \tau-M) .
\end{gathered}
$$

The expressions (5) and (6) coincide with the expressions presented in [13] by Cahn for the indexing of the icosahedral QC diffraction patterns using two integers $(N, M)$. Similar results have been obtained in the papers [10-12] for the octagonal, decagonal, and dodecagonal QC reciprocal lattice indexing. Mentioned QC's are periodic along the highest order symmetry axis; hence their powder diffraction patterns can be indexed using three integers $(N, M, L)$. Obviously, such indexing technique is also valid for application to the flat quasiperiodic structures with a four-fold symmetry examined in this work, in an assumption of their periodicity along the four-fold axis.

It is also necessary to mention that the proposed method of flat quasiperiodic lattice with a four-fold symmetry axis can also be extrapolated on a three-dimensional space. Such structure would be quasiperiodic in all three dimensions but would not contain any forbidden symmetries.

\section{Conclusions}

It is shown that quasilattice modeling using recurrent generation of a lattice point group can be applied not only to the conventional QC, but also to the structures with the 
symmetry axis allowed in the regular crystalline structures. The number $\tau$ can be used as a scaling factor for generating flat quasilattices with the four-fold symmetry axis, which is the same as for icosahedral and decagonal QC modeling.

It is established that the proposed quasiperiodic lattice modeling method does not contradict conventional higher dimensional projection approach and can be used for the analysis of the aperiodic crystal structures.

It is shown that powder diffraction patterns obtained from aperiodic crystals can be indexed using three integer indices. Such an indexing is an extrapolation of the indexing method proposed by Cahn for icosahedral quasicrystals.

\section{References}

1. Oxborrow, M. Random square-triangle tilings: A model for twelvefoldsymmetric quasicrystals / M. Oxborrow, C.L. Henley / Phys. Rev. B. - 1993. - Vol. 48. P. $6966-6998$.

2. Burkov, S.E. Enforcement of matching rules by chemical ordering in the decagonal AlCuCo quasicrystal / S.E. Burkov / Phys. Rev. B. - 1993. - Vol. 47. P. $12325-12328$.

3. Yamamoto, A. Crystallography of Quasiperiodic Crystals / A. Yamamoto / Acta Crystallogr. - 1996. - Vol. A52, Part. 4. - P. $509-560$.

4. Ishimasa, T. Dodecagonal Quasicrystals Still in Progress / T. Ishimasa // Isr. J. Chem. - 2011. - Vol. 51. - P.1216 - 1225

5. Cervellino, A. Structure solution of the basic decagonal Al-Co-Ni phase by the atomic surfaces modelling method / A. Cervellino, T. Haibach, W. Steurer // Acta Crystallogr. - 2002. - Vol. B58, Part. 1. - P. 8 - 33.

6. Yamamoto, A. Six-dimensional model of icosahedral Al-Pd-Mn quasicrystals / A. Yamamoto, H. Takakura, A.P. Tsai / Phys. Rev. B. - 2003. - Vol. 68, Issue 9. P. 094201.

7. Steurer, W. Crystallography of Quasicrystals: Concepts, Methods, and Structures / W. Steurer, S. Deloudi. - Berlin: Springer-Verlag, 2009. - 384 p.

8. Grimm, U. Aperiodic crystals and beyond / U. Grimm / Acta Crystallogr. -2015. - Vol. B71, Part. 3. - P. 258 - 274.

9. Madison, A.E. Simmetriya kvazikristallov / A.E. Madison / Fizika tverdogo tela. -2013. - Vol. 55, No 4. - S. $784-796$.

10. Girzhon, V.V. Modeling of decagonal quasicrystal lattice / V.V. Girzhon, V.M. Kovalyova, O.V. Smolyakov, M.I. Zakharenko / Journal of Non-Crystalline Solids. - 2012. - Vol. 358. - P. $137-144$.

11. Girzhon, V.V. Modeling quasi-lattice with octagonal symmetry / V.V. Girzhon, O.V. Smolyakov, M.I. Zakharenko / Zhurnal Eksperimentalnoy i Teoreticheskoy Fiziki. 2014. - Vol. 146, No. 5(11). - P. 973 - 979.

12. Smolyakov, O.V. Modelirovaniye dodekagonalnyh kvazikristallicheskih reshetok / O.V. Smolyakov, V.V. Girzhon / Zhurnal Eksperimentalnoy i Teoreticheskoy Fiziki. - 2017. - Vol. 152, No. 3(9). - P. 521 - 529.

13. Cahn J.W. Indexing of icosahedral quasiperiodic crystals / J.W. Cahn, D. Shechtman, D. Gratias / Journal of Materials Research. - 1986. - Vol.1, Issue 1. P. $13-26$. 